\title{
RNA-binding motif protein 5 inhibits the proliferation of cigarette smoke-transformed BEAS-2B cells through cell cycle arrest and apoptosis
}

\author{
XUE-JIAO LV ${ }^{1}$, YAN-WEI DU ${ }^{2}$, YU-QIU HAO ${ }^{1}$, ZHEN-ZHONG SU ${ }^{1}$, \\ LIN ZHANG $^{1}$, LI-JING ZHAO ${ }^{3}$ and JIE ZHANG ${ }^{1}$ \\ ${ }^{1}$ Department of Respiratory Medicine, The Second Affiliated Hospital of Jilin University, Changchun, Jilin 130041; \\ ${ }^{2}$ Department of Pathology, College of Basic Medicine, Changchun University of Chinese Medicine, \\ Changchun, Jilin 130117; ${ }^{3}$ Department of Pathophysiology, College of Basic Medicine, \\ Jilin University, Changchun, Jilin 130021, P.R. China
}

Received October 14, 2015; Accepted December 11, 2015

DOI: $10.3892 / o r .2016 .4551$

\begin{abstract}
Cigarette smoking has been shown to be the most significant risk factor for lung cancer. Recent studies have also indicated that RNA-binding motif protein 5 (RBM5) can modulate apoptosis and suppress tumor growth. The present study focused on the role of RBM5 in the regulation of cigarette smoke extract (CSE)-induced transformation of bronchial epithelial cells into the cancerous phenotype and its mechanism of action. Herein, we exposed normal BEAS-2B cells for 8 days to varying concentrations of CSE or dimethylsulfoxide (DMSO), followed by a recovery period of 2 weeks. Next, the RBM5 protein was overexpressed in these transformed BEAS-2B cells though lentiviral infection. Later, the morphological changes, cell proliferation, cell cycle, apoptosis, invasion and migration were assessed. In addition, we analyzed the role of RBM5 in xenograft growth. The expression of RBM5 along with the genes related to cell cycle regulation, apoptosis and invasion were also examined. Finally, our results revealed that BEAS-2B cells exposed to $100 \mu \mathrm{g} / \mathrm{ml}$ CSE acquired phenotypic changes and formed tumors in nude mice, indicative of their cancerous transformation and had reduced RBM5 expression. Subsequent overexpression of RBM5 in these cells significantly inhibited their proliferation, induced G1/S arrest, triggered apoptosis and inhibited their invasion and migration, including xenograft growth. Thus, we established an in vitro model of CSE-induced cancerous transformation and concluded that RBM5 overexpression inhibited the growth of these transformed cells through cell cycle arrest and induction of apoptosis. Therefore, our study
\end{abstract}

Correspondence to: Dr Jie Zhang, Department of Respiratory Medicine, The Second Affiliated Hospital of Jilin University, 218 Ziqiang Street, Nanguan, Changchun, Jilin 130041, P.R. China E-mail: doctorzhangj@sina.com

Key words: RBM5, cigarette smoke extract, BEAS-2B cells, cancerous transformation, lung cancer suggests the importance of RBM5 in the pathogenesis of smoking-related cancer.

\section{Introduction}

Cigarette smoking is not only one of the most severe public health issues but is also one of the most significant etiological factors that contribute to the development of lung cancer, chronic obstructive pulmonary disease, interstitial lung diseases and bronchial asthma (1). Based on the reports of the World Health Organization, smoking will cause roughly 10 million deaths per annum by the year 2030 (2). Approximately $25 \%$ of these deaths will be exclusively from lung cancer. Cigarette smoking has been established as the most significant risk factor for lung cancer, and individuals who smoke usually have a 15- to 30-fold increased risk of developing lung cancer (3). Current treatment options are inadequate due to the poor understanding of the molecular basis of cigarette-induced malignant transformation of bronchial epithelial cells and the subsequent development of lung cancer.

The 'loss of function' of tumor-suppressor genes appears to be a critical step in the pathogenesis of human lung cancer. The gene for RNA-binding motif protein 5 (RBM5), also known as LUCA-15 or H37, is mapped to human chromosome $3 p 21.3$. This region of the human chromosome is deleted in a large number of human cancers and is presumed to contain one or more tumor-suppressor genes (4). Previous studies have shown that RBM5 is downregulated in many different types of cancers, including ras-transformed Rat-1 embryonic fibroblastic cells (5), breast (1) and prostate cancer (6), human vestibular schwannoma (7) and primary lung cancer (8). It was reported that expression of RBM5 mRNA is marginally upregulated whereas the protein was significantly upregulated in breast tumor tissues compared to that in non-tumor tissues. These data revealed that RBM5-related factors are differentially expressed in breast cancer and suggest the involvement of complex inter-related regulatory networks involving alternative splicing, oncogenic expression and tissue-specific function (9-11). 
RBM5, through pre-mRNA splicing of multiple target genes, has the ability to modulate apoptosis and cell cycle arrest in several types of malignancies, particularly non-small cell lung cancer cells $(4,5,8,12-18)$. In addition, the loss of RBM5 protein expression in primary lung tumors correlates with increased lymph node metastasis (13). RBM5 expression is also negatively correlated with the smoking status in patients with lung cancer (12). Cigarette smoking directly affects human bronchial epithelial cells, which happen to be the major component of the airway epithelium. However, the effect of cigarette smoking on RBM5 expression in bronchial epithelial cells is not clear; in addition, it would be important to determine whether this cigarette smoke-induced cancerous transformation of bronchial epithelial cells involves RBM5. Thus, by developing an appropriate in vitro model, we attempted to address this issue and to elucidate the role of RBM5 in the transformation of bronchial epithelial cells.

\section{Materials and methods}

Cell culture. Human bronchial epithelial BEAS-2B cells were purchased from the American Type Culture Collection (ATCC; Manassas, VA, USA). The cells are non-tumorigenic in nude mice. BEAS-2B cells were maintained in RPMI-1640 medium (Gibco-BRL, Carlsbad, CA, USA) containing 10\% fetal bovine serum (FBS), $100 \mathrm{U} / \mathrm{ml}$ penicillin and $0.1 \mathrm{mg} / \mathrm{ml}$ streptomycin [penicillin and streptomycin solution (100X)] (both from Gibco, Waltham, MA, USA) at $37^{\circ} \mathrm{C}$ in a humidified atmosphere containing $5 \% \mathrm{CO}_{2}$.

Preparation of cigarette smoke extract (CSE). The 100\% CSE was generated as previously described (19). Briefly, cigarette smoke was prepared from research-grade cigarettes obtained from the Tobacco Research and Development Center of the University of Kentucky, USA. A syringe-driven apparatus device was designed and operated to allow a stream of smoke to flow into a tube-shaped trap. The smoke then entered a flask submerged in liquid nitrogen. The amount of smoke obtained was calculated by the increase in the weight inside the flask. The smoke particulates were collected in dimethylsulfoxide (DMSO) at a concentration of $40 \mathrm{mg} / \mathrm{ml}$ and were sterilefiltered through a $0.22-\mu \mathrm{m}$-pore filter (Millipore, Watford, $\mathrm{UK}$ ), aliquoted and then stored at $-80^{\circ} \mathrm{C}$, for use in subsequent experiments.

Cell proliferation assay. Exponentially growing BEAS-2B cells were exposed to varying concentrations $(0,25,50$ or $100 \mu \mathrm{g} / \mathrm{ml}$ ) of CSE or DMSO (CON) for 8 days, and fresh CSE was added each day. After exposure, the cells were washed twice with phosphate-buffered saline (PBS) and then allowed to recover for a period of 2 weeks. In this way, we generated five cell populations for each concentration of CSE. After exposure to CSE, the proliferative changes of five cell populations were examined by the 3-(4,5-dimethylthiazol-2-yl)-2,5-diphenyltetrazolium bromide (MTT) assay. Briefly, the cells $\left(5 \times 10^{3}\right)$ were cultured in each well of 96 -well plates for $72 \mathrm{~h}$. During the last $4 \mathrm{~h}$ of incubation, the cells were exposed to $20 \mu \mathrm{l} /$ well of $5 \mathrm{mg} /$ ml MTT solution (Sigma-Aldrich, St. Louis, MO, USA). The resulting formazan was dissolved in $150 \mu \mathrm{l}$ of DMSO, and the absorbance was measured at $490 \mathrm{~nm}$ using a SpectraMax 190 microplate reader (Molecular Devices, Sunnyvale, CA, USA). Similarly, the cell viability was measured using the cell viability assay. In addition, the morphology of each individual group of cells was examined under a phase contrast microscope (CKX41; Olympus, Japan).

Cell invasion and migration assays. The invasion and migration of the cells were determined by Transwell invasion and wound-healing assays. Briefly, the cells $\left(5 \times 10^{3}\right)$ were cultured in FBS-free RPMI-1640 medium in the top chambers of 24-well Transwell plates $(0.4 \mu \mathrm{m}$; Corning Costar, Cambridge, MA, USA) coated with Matrigel (BD Biosciences). The lower chambers were filled with RPMI-1640 supplemented with $10 \%$ FBS as a chemoattractant for $72 \mathrm{~h}$. The cells that had invaded to the bottom of the top chamber were stained with crystal violet stain dissolved in $2 \%$ ethanol, and at least 10 different fields were counted under a phase contrast microscope to calculate the invasion efficiency.

For the wound-healing assay, the cells $\left(1 \times 10^{5}\right)$ were first starved in $2 \%$ FBS medium in 24-well plates overnight. The next day, a scratch was created in the cell layer with the small tip of a ruler. The scratched areas were washed with PBS to remove free cells, and the adherent cells were cultured in complete medium for one more week. This procedure was followed by longitudinal photo imaging under a phase contrast microscope.

Western blot analysis. After treatment, the cultured cells were lysed using RIPA lysis buffer. The protein concentration was determined using a Bio-Rad protein assay kit (Bio-Rad), and protein samples $(40 \mu \mathrm{g})$ were separated on a $10 \%$ sodium dodecyl sulfate-polyacrylamide gel and transferred onto a polyvinylidene difluoride membrane. The membranes were then blocked with 5\% non-fat dry milk in Tris-buffered saline for $2 \mathrm{~h}$, followed by incubation with primary antibodies, including anti-RBM5 (ab85504), anti-cyclin A (ab80792), anti-cyclin D1 (ab7958), anti-cyclin-dependent kinase 4 (CDK4) (EPR4513, ab108357), anti-CDK6 (EPR4515, ab124821), anti-p53 (EPR17343, ab174977), anti-p21 (ab18209), anti-matrix metalloproteinase (MMP)-2 (ab110168), anti-MMP-9 (ab5707), anti-hypoxia-inducible factor (HIF)-1 $\alpha$ (ab10363), anti-vascular endothelial growth factor (VEGF) (ab46154), anti-K-ras (ab102007), anti-C-myc (ab39688), antiBax (E63, ab32503), anti-bcl-2 (ab117115) and anti- $\beta$-actin (E247, ab32572) from Abcam (Cambridge, MA, USA) as well as anti-cleaved-caspase-3 (\#9661) and anti-cleaved-caspase-9 (\#9509) from Cell Signaling Technology (Beverly, MA, USA), at 1:500 dilutions for $2 \mathrm{~h}$ at room temperature. The membranes were then washed three times with $0.1 \%$ Tween-20/PBS prior to incubation with the appropriate secondary antibody conjugated with peroxidase (anti-rabbit or anti-mouse IgG) (Santa Cruz Biotechnology) for 1.5 h. Finally, the proteins were detected using the enhanced chemiluminescence detection reagent (Millipore, Billerica, MA, USA), and their levels were quantified by densitometry using Quantity One software (Bio-Rad Laboratories).

Reverse transcription-polymerase chain reaction (RT-PCR) analysis. Total RNA was extracted using TRIzol solution (Takara Biotechnology Co., Dalian, China), according to the 
Table I. List of primer sequences used for qPCR analyses.

\begin{tabular}{lll}
\hline Gene & \multicolumn{1}{c}{ Forward primer } & \multicolumn{1}{c}{ Reverse primer } \\
\hline GAPDH & 5'-AGAAGGCTGGGGCTCATTTG-3' & 5'-AGGGGCCATCCACAGTCTTC-3' \\
p53 & 5'-CCATCTACAAGCAGTCACAG-3' & 5'-CAAATCTACAAGCAGTCACAG-3' \\
p21 & 5'-ACTTCGACTTTGTCACCGAGA-3' & 5'-GAGGCACAAGGGTACAAGACA-3' \\
MMP-9 & 5'-CGAACTTCGACACTGACAAGAAGT-3' & 5'-GCACGCTGGAATGATCTAAGC-3' \\
MMP-2 & 5'-CTGGGTTTACCCCCTGATGTCC-3' & 5'-AACCGGGGTCCATTTTCTTCTTT-3' \\
Caspase-3 & 5'-GAAACCTCCGTGGATTCAAA-3' & 5'-AGCCCATTTCAGGGTAATCC-3' \\
Caspase-9 & 5'-TCCTGGTACATCGAGACCTTG-3' & 5'-AAGTCCCTTTCGCAGAAACAG-3' \\
Bax & 5'-ACCAAGAAGCTGAGCGAGTG-3' & 5'-CCCAGTTGAAGTTGCCATCA-3' \\
Bcl-2 & 5'-ACGACTTCTCCCGCCGCTAC-3' & 5'-CCCAGCCTCCGTTATCCTG-3' \\
Cyclin A & 5'-TGGACCTTCACCAGACCTAC-3' & 5'-GGTTGAGGAGAGAAACACCA-3' \\
Cyclin D1 & 5'-AGTTGCTGCAAATGGAACTG-3' & 5'-AAAGGTCTGTGCATGTTTGC-3' \\
CDK4 & 5'-TTTGATCTCATTGGATTGCC-3' & 5'-AGGTCAGCATTTCCAGCAG-3' \\
CDK6 & 5'-TGGAGTGTTGGCTGCATATT-3' & 5'-ACAGGGCACTGTAGGCAGAT-3' \\
HIF-1 $\alpha$ & 5'-CCACAGGACAGTACAGGATG-3' & 5'-TCAAGTCGTGCTGAATAATACC-3' \\
VEGF & 5'-AAACCCTGAGGGAGGCTC-3' & 5'-TACTTGCAGATGTGACAAGCCG-3' \\
K-ras & 5'-GGGGAGGGCTTTCTTTGTGTA-3' & 5'-GTCCTGAGCCTGTTTTGTGTC-3' \\
C-myc & 5'-ACAGCTACGGAACTCTTGTGCGTA-3' & 5'-GCCCAAAGTCCAATTTGAGGCAGT-3' \\
RBM5 & 5'-ACACGATGGATGGAAGCCA-3' & 5'-TCTGCTCTGCCTCTGACTT-3'
\end{tabular}

GAPDH, glyceraldehyde-3-phosphate dehydrogenase; MMP, matrix metalloproteinase; RBM5, RNA-binding motif protein 5; HIF-1 $\alpha$, hypoxia-inducible factor $1 \alpha$; VEGF, vascular endothelial growth factor.

manufacturer's instructions. cDNAs were reverse transcribed using a reverse transcription kit (Takara Biotechnology), according to the manufacturer's instructions. Semi-quantitative real-time PCR was then performed using the following primers: RBM5 forward, 5'-ACACGATGGATGGAAGCCA-3' and reverse, 5'-TCTGCTCTGCCTCTGACTT-3'; glyceraldehyde-3-phosphate dehydrogenase (GAPDH) forward, 5'-GGG TGATGCTGGTGCTGAGTATGT-3' and reverse, 5'-AAGAA TGGGAGTTGCTGTTGAAGTC-3'. These oligonucleotides were synthesized by Shanghai Promega Biological Products, Ltd. (Shanghai, China). The relative levels of target gene mRNA transcripts to the control GAPDH were determined by quantitative RT-PCR (qRT-PCR) on a Stratagene Mx3000P Real-Time PCR system (Stratagene, La Jolla, CA, USA) using the FastStart Universal SYBR-Green Master reagent (Roche, Mannheim, Germany) and specific primers (Table I). The relative levels of target gene mRNA transcripts to the control GAPDH were calculated by the formula: $2^{-\Delta \Delta \mathrm{Ct}}$.

Lentiviral vector construction and infection. Lentiviral vectors conjugated to green fluorescent protein (GFP) were used to achieve high efficiency of infection and subsequent stable expression of RBM5 protein in the target cells. Recombinant pGC-LV-GV287-GFP vector with either the RBM5 (NM_005778) gene (LV-RBM5) or the scrambled control sequence (LV-GV287) was constructed by GeneChem Co. (Shanghai, China). Next, the cells $\left(5 \times 10^{5}\right)$ were seeded in a 6 -well culture plate and were incubated for $12 \mathrm{~h}$ to reach $30 \%$ confluency. Later, these cells were infected with LV-RBM5 (RBM5 overexpression group) or LV-GV287 (negative control group) lentiviral particles at a multiplicity of infection (MOI) of 20 plaque-forming units/cell. The plates were then incubated for $24 \mathrm{~h}$ prior to changing the medium to fresh virus-free medium. Three days later, the GFP expression was assessed in the target cells to evaluate the efficiency of infection, and the cells were harvested for western blotting and RT-PCR analyses.

Colony formation assay. The cells were digested with $0.25 \%$ trypsin (Gibco-BRL, Grand Island, NY, USA) to obtain a single-cell suspension with a density of $0.1 \times 10^{6}$ cells $/ \mathrm{ml}$. The cell suspensions were then transferred into 6-well plates (300 cells/well) and incubated at $37^{\circ} \mathrm{C}$ in an atmosphere of $5 \%$ $\mathrm{CO}_{2}$ for 21 days. The formed cell colonies were stained with crystal violet dye in $50 \%$ methanol and $10 \%$ acetic acid, and the stained colonies that had $>50$ cells were counted in a blinded manner. The colony formation efficiency was calculated as the ratio of the colony number to the plated cell number.

Flow cytometric analysis. Cells were trypsinized, washed twice with ice-cold PBS, fixed in $70 \%$ ethanol and stained with propidium iodide (PI; $5 \mu \mathrm{g} / \mathrm{ml} \mathrm{PI}$ in PBS containing $0.1 \%$ Triton X-100 and $0.2 \mathrm{mg} / \mathrm{ml}$ RNase A) overnight at $4^{\circ} \mathrm{C}$ in the dark. The distribution of different cell phases was analyzed by flow cytometry using ModFit LT software.

Furthermore, the harvested cells were stained with Annexin V-PE and 7-AAD. The percentages of apoptotic cells were analyzed by flow cytometric analysis.

Tumorigenic and histological studies. BALB/c athymic nude male mice (nu/nu; 5 weeks) were purchased from the Institute of Zoology, Chinese Academy of Sciences, Beijing, China. The 
care and use of the animals was in accordance with the Animal Care guidelines, and the protocol was approved by Jilin University, Animal Care Committee. The cells were washed and resuspended in PBS. A total of $5 \times 10^{6}$ cells suspended in a $150-\mu 1$ volume were subcutaneously injected into the right flanks of each nude mouse. The size of the tumors was measured using a caliper, starting on day 7 after cell injection. The animals were palpated for tumor appearance once a week and were sacrificed as soon as the tumor nodules reached $1.0 \mathrm{~cm}$ in size. Xenograft tissues were immediately harvested after euthanasia, fixed in neutral buffered formalin, and embedded in paraffin for routine histological evaluation by staining $5-\mu$ m-thick sections with hematoxylin and eosin (H\&E) stain.

Statistical analysis. All experiments were performed at least in triplicate, and the data are presented as means \pm standard deviation (SD). Statistical significance was determined by analysis of the t-test using SPSS version 17.0 (SPSS, Inc., Chicago, IL, USA) software. A P-value of $<0.05$ was considered to indicate a statistically significant result.

\section{Results}

CSE exposure promotes the cancerous transformation of normal human bronchial epithelial cells. We evaluated the effect of CSE on non-cancerous human bronchial epithelial BEAS-2B cells by exposure to varying concentrations of CSE $(0,25,50$ and $100 \mu \mathrm{g} / \mathrm{ml}$, respectively) or DMSO (CON) for 8 days, followed by a recovery period of 2 weeks. The results of the MTT assay showed that the cell population, which survived the toxic effects of CSE for 8 days, acquired phenotypic changes after a recovery period of 2 weeks, including enhanced cell proliferation (Fig. 1A) and shorter doubling times during the recovery period.

In addition, we analyzed whether prolonged exposure to CSE led to the cancerous transformation of non-tumorous epithelial BEAS-2B cells, as they reflected an enhanced cell proliferation. Therefore, to determine whether this transformation occurred, we characterized the morphological changes in BEAS-2B cells, longitudinally. During the recovery period after CSE exposure, the cells treated with $100 \mu \mathrm{g} / \mathrm{ml}$ CSE showed aberrant and condensed nuclei in addition to abnormal nuclear cytoplasmic ratios, compared to the control cells (Fig. 1B). Similar changes were also observed for the cells treated with 25 and $50 \mu \mathrm{g} / \mathrm{ml} \mathrm{CSE}$, but the effects were not as obvious as with the higher dose $(100 \mu \mathrm{g} / \mathrm{ml})$ of CSE. Moreover, treatment with $100 \mu \mathrm{g} / \mathrm{ml} \mathrm{CSE}$ also revealed multiple layers of cell growth, indicative of the loss of contact inhibition. These data thus indicate that exposure to CSE promoted the cancerous transformation of BEAS-2B cells.

CSE-transformed BEAS-2B cells exhibit enhanced invasion and migration ability. The invasion and migration of the CSE-transformed BEAS-2B cells were determined by Transwell invasion and wound-healing assays. As shown in Fig. 1C and D, CSE exposure $(25,50$ and $100 \mu \mathrm{g} / \mathrm{ml})$ or DMSO increased the ability of these cells to invade the extracellular matrix, compared to the control treatment. This effect was most significant $(\mathrm{P}<0.01)$ following exposure to $100 \mu \mathrm{g} / \mathrm{ml} \mathrm{CSE}$. Similarly, the wound-healing assay results also showed that
CSE exposure enhanced the migration of CSE-transformed cells compared to the control group (Fig. 1E).

Prolonged exposure of CSE induces the expression of oncogenes and xenograft growth. Next, we examined the protein and mRNA levels of various oncogenes, including K-ras, c-myc and cyclin A and cyclin D1, in the CSE-transformed BEAS-2B cells. Western blot analysis showed that the expression of these proteins was significantly enhanced in the CSE-transformed BEAS-2B cells treated with varying concentrations of CSE $(\mathrm{P}<0.01)$ (Fig. 1F and $\mathrm{G})$. In addition, the $\mathrm{qPCR}$ results demonstrated a similarly significant $(\mathrm{P}<0.05)$ increase in the expression of K-ras, c-myc, cyclin A and cyclin D1 mRNA levels in the CSE-transformed BEAS-2B cells, compared with the control group (Fig. $1 \mathrm{H}$ ). These results indicate that CSE regulated the expression of these oncogenes at the transcriptional level in the CSE-transformed BEAS-2B cells.

Subsequently, we analyzed the ability of the CSE-transformed BEAS-2B cells to induce xenograft growth. BEAS-2B cells were treated with varying concentrations $(0,25,50$ or $100 \mu \mathrm{g} / \mathrm{ml}$ ) of CSE or DMSO (CON) for 8 days, and then allowed to recover for a period of 2 weeks. Next, we implanted BEAS-2B cells, BEAS-2B cells treated with DMSO (CON cells) or CSE-transformed BEAS-2B cells into immunodeficient mice (5 nude mice/cell line) and observed that the BEAS-2B and CON cells did not form tumors, even after 3 months of injection, while all mice $(n=5)$ implanted with the transformed BEAS-2B cells exposed to $100 \mu \mathrm{g} / \mathrm{ml}$ CSE developed tumors with a latency period of $\sim 2$ weeks, indicating a high efficiency of murine tumorigenicity (Fig. 1I). However, the transformed cells exposed to 25 or $50 \mu \mathrm{g} / \mathrm{ml} \mathrm{CSE} \mathrm{did} \mathrm{not}$ form tumors. The histological examination further confirmed that the cells exposed to $100 \mu \mathrm{g} / \mathrm{ml}$ CSE formed carcinomas, revealing that the tumors were composed of variably sized nests of polyhedral cells that were connected by thin stromal strands. These tumor cells were moderately pleiomorphic, had shapes from round to elongated, and had prominent multiple nuclei (Fig. 1I). We designated this CSE-induced cancerous transformation of BEAS-2B cells as 'T3 cells'.

CSE-transformed BEAS-2B cells have reduced expression of $R B M 5$. To analyze the role of RBM5 in the cancerous transformation of normal human bronchial epithelial cells, we first determined the expression of RBM5 in these cells exposed to CSE at varying concentrations. We observed that both at the mRNA (qRT-PCR) and protein levels (western blotting), the RBM5 expression was reduced with an increasing CSE concentration, compared to the control group. The fold-change in the gene expression was significantly reduced in the T3 cells (cells treated with $100 \mu \mathrm{g} / \mathrm{ml}$ CSE) $(\mathrm{P}<0.01)$ (Fig. 2A-C). It is important to mention that we also analyzed the expression of RBM5 in A549 cells, which are inherently cancerous cells, as a control and observed that RBM5 expression in these cells was also less than that in the control cells.

Analysis of RBM5 overexpression in the CSE-transformed $B E A S-2 B$ cells infected with lentiviral vectors. The T3 cells were successfully infected with recombinant lentiviral vectors expressing GFP, LV-RBM5 or vector alone (LV-GV287). Using fluorescence microscopy, the GFP expression was analyzed 

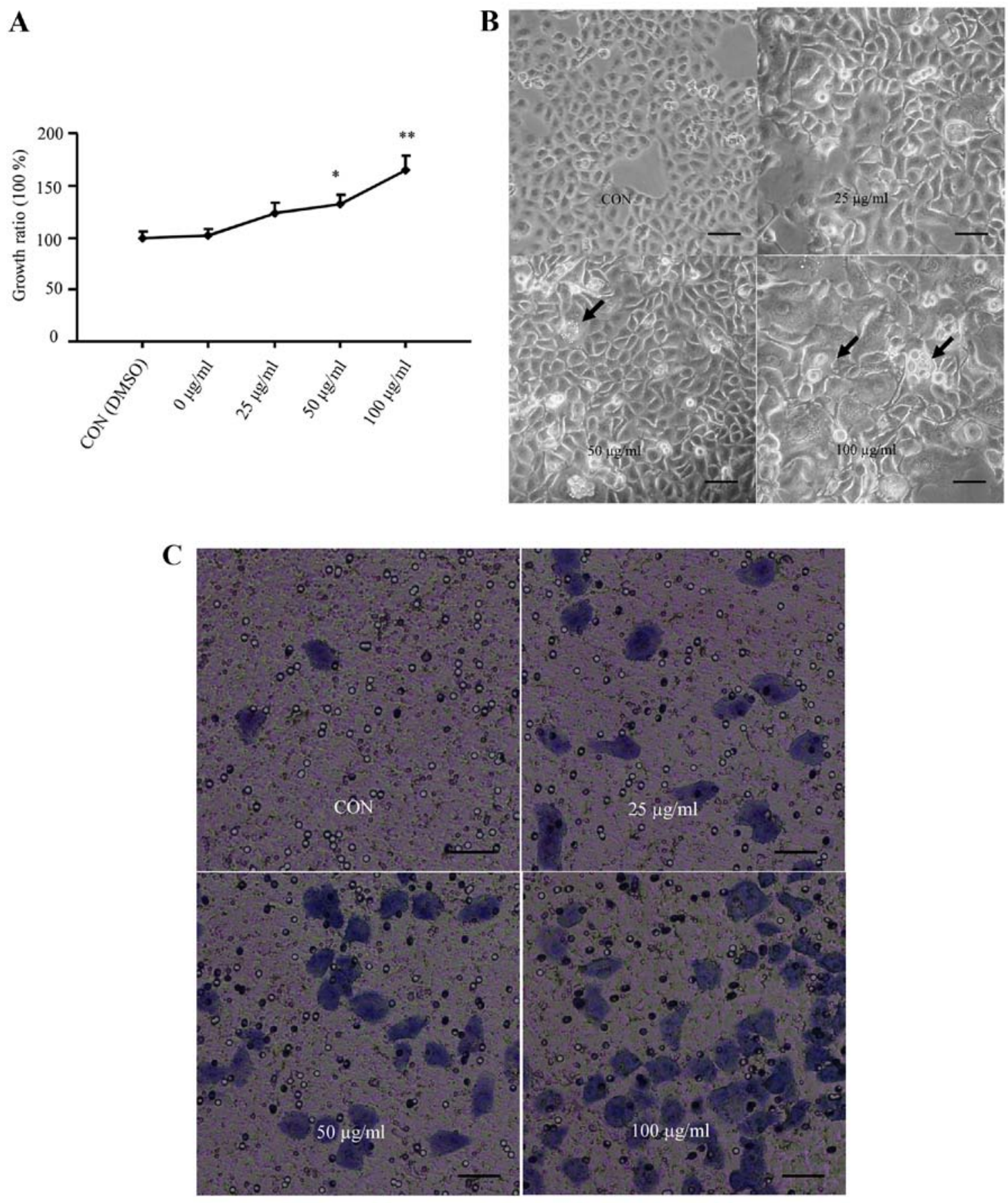

D

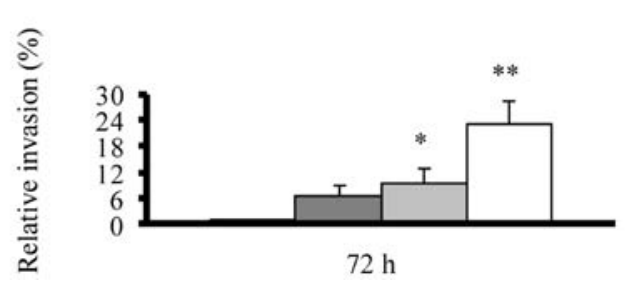

- Control

口 $25 \mu \mathrm{g} / \mathrm{ml}$

ㅁ $50 \mu \mathrm{g} / \mathrm{ml}$

$\square 100 \mu \mathrm{g} / \mathrm{ml}$

Figure 1. Analysis of the effect of CSE on normal human bronchial epithelial cells. (A) The effects of CSE on the growth rates of BEAS-2B cells as determined by the MTT assay. The growth rates of control cells without CSE exposure were designated as $0 \%$. (B) Morphological changes in the BEAS-2B cells following prolonged CSE exposure at varying concentrations. The images were captured under a phase contrast light microscope. Scale bar, $50 \mu \mathrm{m}$. (C and D) The invasive ability of the CSE-transformed BEAS-2B cells as determined by the Transwell invasion assay. Scale bar, $20 \mu \mathrm{m} .{ }^{*} \mathrm{P}<0.05,{ }^{* *} \mathrm{P}<0.01 \mathrm{vs}$. the control.

3 days after the infection, and it was observed that the efficiency of the infection was $\sim 80 \%$ at an MOI of 20 (Fig. 3A). Therefore, an MOI of 20 was used for all other infection experiments in the present study. The RT-PCR analysis demonstrated a significant $(\mathrm{P}<0.01)$ increase in the expression level of RBM5 mRNA in the T3-LV-RBM5 group, compared 

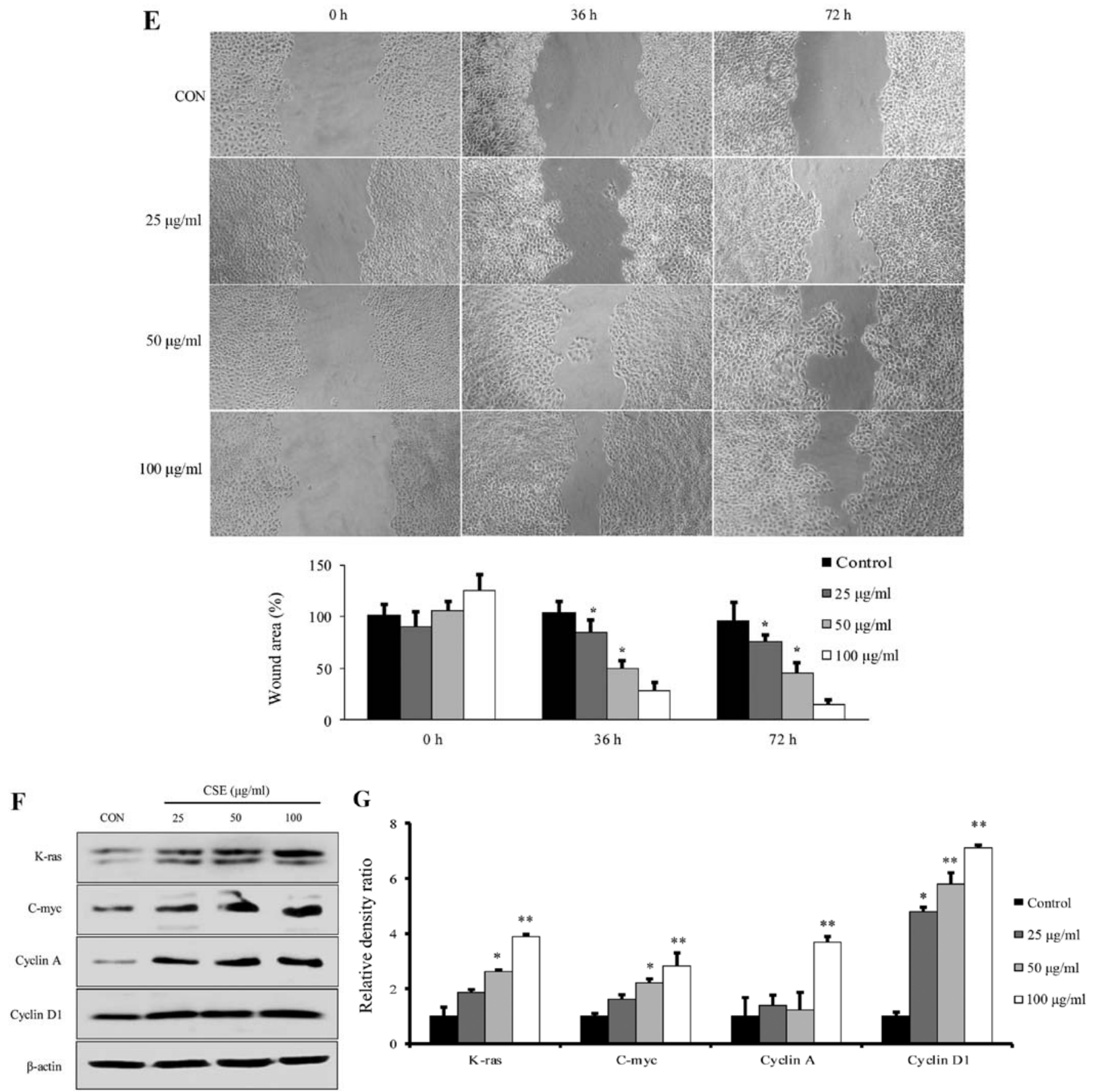

Figure 1. Continued. (E) The migration ability of the CSE-transformed BEAS-2B cells exposed to varying concentrations of CSE. The images were recorded after 0,36 and $72 \mathrm{~h}$ of exposure. (F) The effects of CSE exposure on the expression of oncogenes, K-ras, c-myc, cyclin A and cyclin D1. The expression of $\beta$-actin was used as a loading control. (G) The normalized expression of these oncogenes. ${ }^{*} \mathrm{P}<0.05,{ }^{* *} \mathrm{P}<0.01$ vs. the control.

with the negative control group (T3-LV-GV287 cells) and the non-transfected control group (T3-CON cells) (Fig. 3B and D). Similarly, western blot analysis also confirmed the overexpression of RBM5 (Fig. 3C and E).

RBM5 overexpression inhibits the proliferation and colony formation of CSE-transformed BEAS-2B cells. Next, we analyzed whether an elevated RBM5 expression has any effect on the cell proliferation and colony formation of CSE-transformed BEAS-2B cells. As shown in Fig. 4A, RBM5 overexpression markedly inhibited the cell growth of the T3 cells overexpressing RBM5, compared to the T3 cells transfected with the empty vector $(\mathrm{P}<0.01)$. Simultaneously, RBM5 overexpression markedly inhibited the cell growth of the A549 cells, compared to the A549 cells transfected with the empty vector, while the growth of BEAS-2B cells was not significantly changed after RBM5 overexpression for 3 days, compared to BEAS-2B cells transfected with the empty vector. Moreover, the ability of these different groups of cells to form colonies was assessed after culturing for an additional 21 days, and images under a microscope were recorded. Quantitative analysis of individual clones with $>50$ cells 


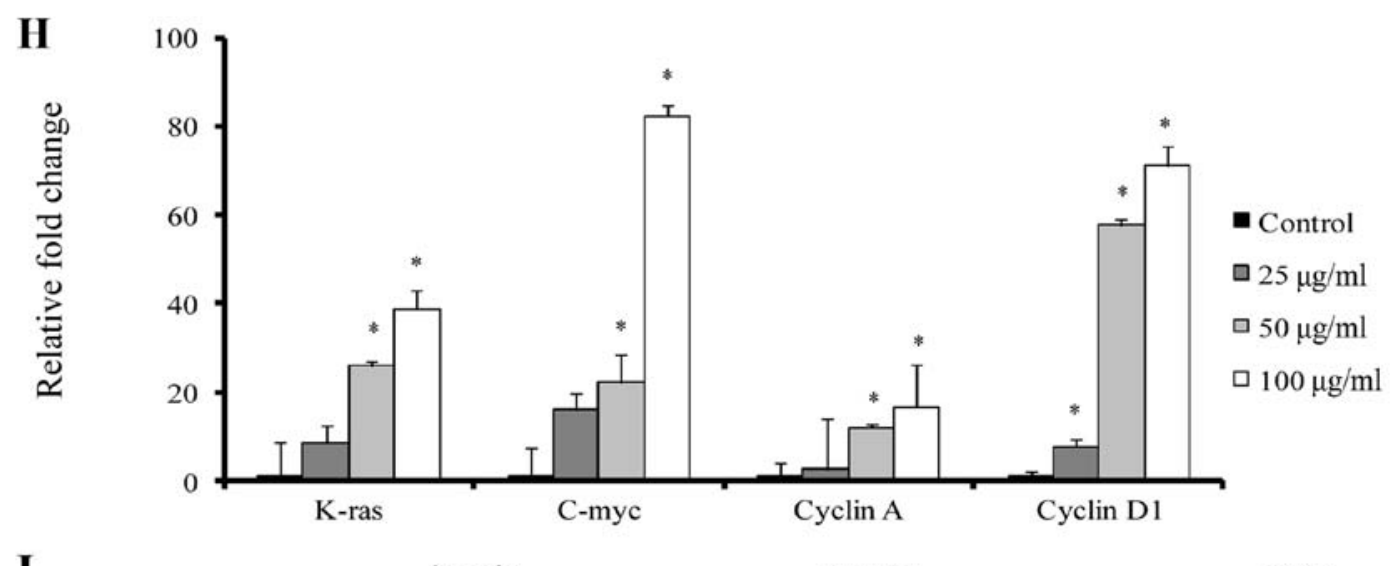

I

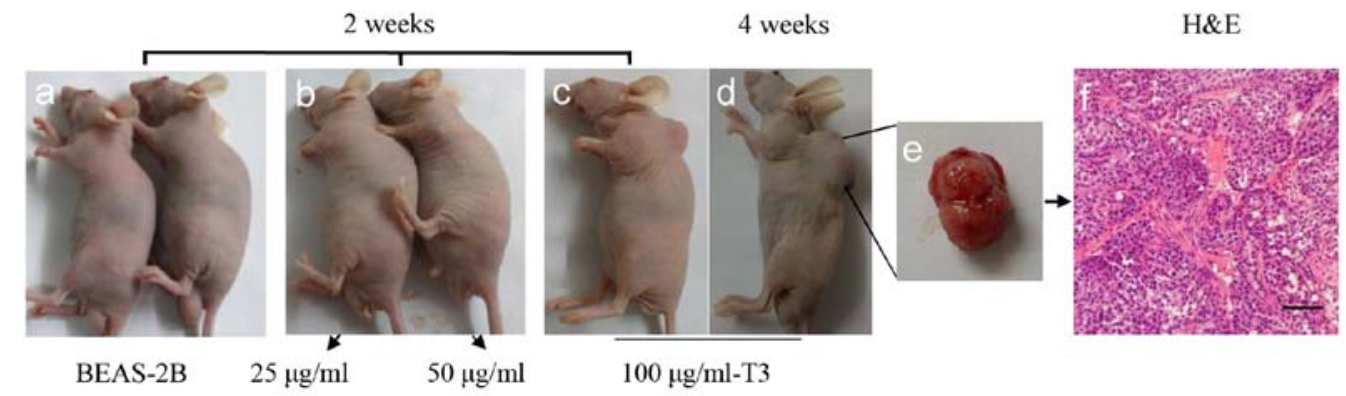

Figure 1. Continued. (H) Relative mRNA expression of the oncogenes by RT-PCR. (I) Xenograft tumor growth at 2 weeks after inoculation with CSEtransformed BEAS-2B cells exposed to different CSE concentrations. The arrows indicate a large tumor mass and a section of a representative tumor stained with H\&E. Scale bar, $50 \mu \mathrm{m}$. The data in all panels were collected from three individual experiments. ${ }^{*} \mathrm{P}<0.05,{ }^{* *} \mathrm{P}<0.01$ vs. the control.

A

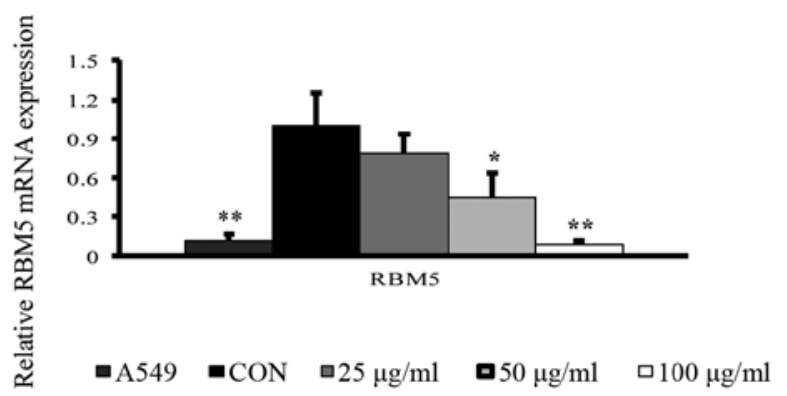

B

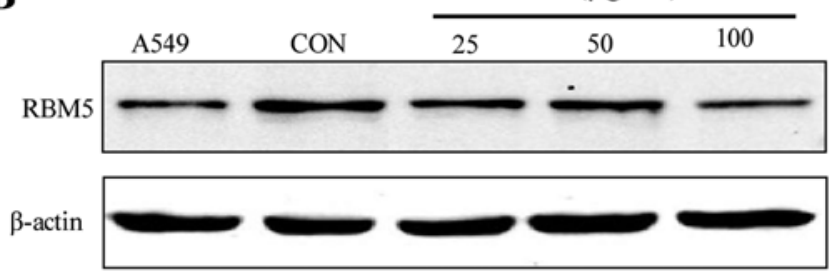

C

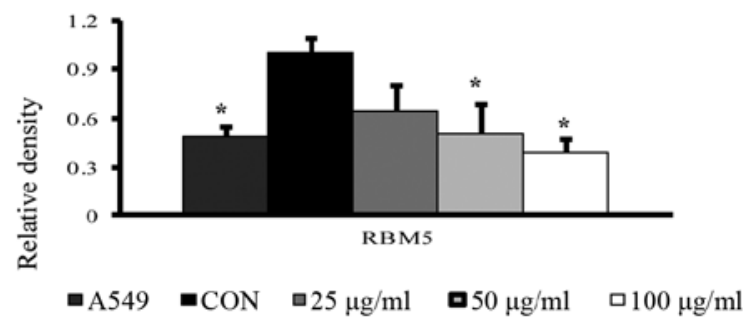

Figure 2. Analysis of RBM5 expression in the CSE-transformed BEAS-2B cells. (A) mRNA expression of RBM5 as analyzed by qRT-PCR in the BEAS-2B cells exposed to different concentrations of CSE for 8 days, followed by a recovery period of 2 weeks. A549 cells were used as a control tumor cell line. GAPDH was used as an internal reference. Similarly, (B and C) RBM5 expression as analyzed by western blot analysis. $\beta$-actin expression was used as a loading control. All data points represent the mean \pm SD from three replicate experiments. " $\mathrm{P}<0.05$, vs. the control.

revealed a significantly $(\mathrm{P}<0.01)$ reduced colony size and fewer numbers of cells in the T3-LV-RBM5 group, compared to the T3-CON and T3-LV-GV287 groups (Fig. 4B and C). This set of data indicated that overexpression of RBM5 inhibited the proliferation and the colony formation potential of the CSE-transformed BEAS-2B cells.

RBM5 overexpression inhibits the invasion and migration of CSE-transformed BEAS-2B cells. We further determined the impact of RBM5 on the invasion and migration ability of CSE-transformed BEAS-2B cells. As shown in Fig. 5A and B, the ability of T3-LV-RBM5 cells to invade through the extracellular matrix was significantly reduced, compared with the T3-CON and T3-LV-GV287 cells. Similarly, the results of the wound-healing assay also showed that compared with the control groups, the migration of the T3-LV-RBM5 cells was markedly decreased (Fig. 5C and D). Furthermore, expression analysis of the genes commonly implicated in cell 


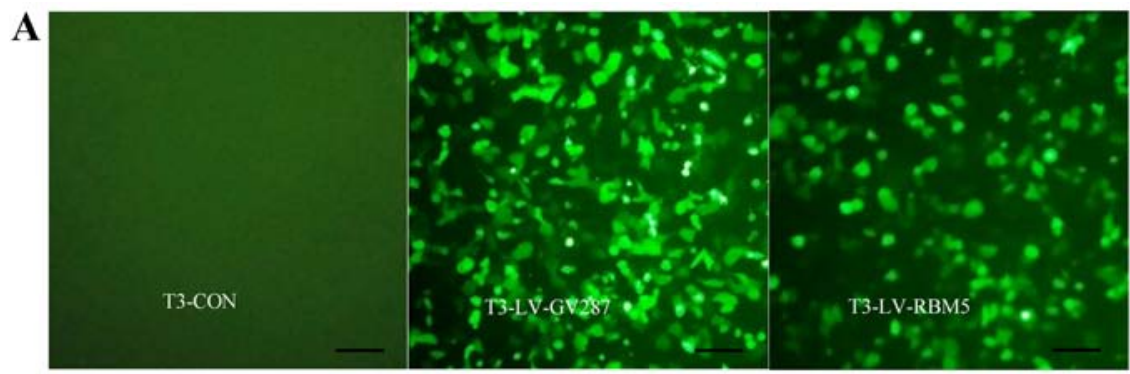

B

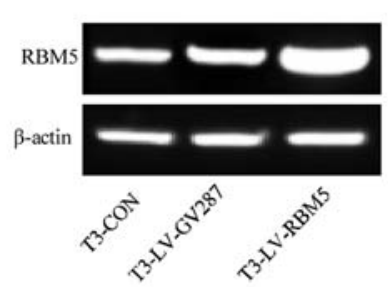

C

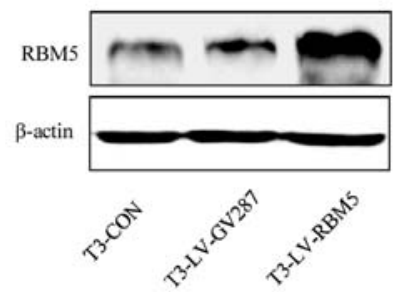

D

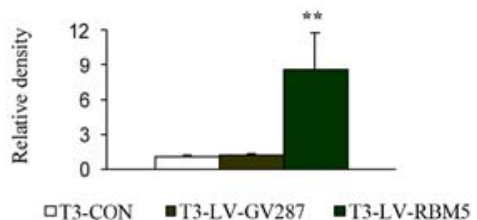

$\mathbf{E}$

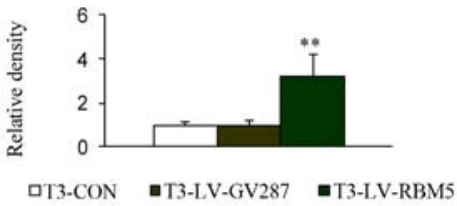

Figure 3. Analysis of CSE-transformed BEAS-2B cells for RBM5 overexpression after infection with a lentiviral vector expressing RBM5. (A) GFP expression, reflecting the infection efficiency in the CSE-transformed BEAS-2B cells (T3 cells). Scale bar, $50 \mu \mathrm{m}$. T3-CON, control group with no transfection; T3-LV-GV287, negative control group transfected with the GFP lentiviral vector LV-GV287; and T3-LV-RBM5, RBM5 overexpression group transfected with the GFP lentiviral vector LV-RBM5. (B and D) Relative RBM5 mRNA levels. (C and E) RBM5 protein expression in the T3 cells after lentiviral infection. GAPDH and $\beta$-actin were used as internal controls. Data shown are means \pm SD of three separate experiments. ${ }^{* *} \mathrm{P}<0.01$, vs. the control.

A

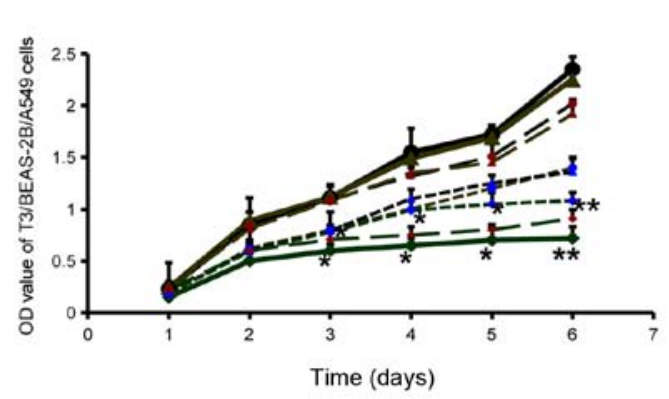

B

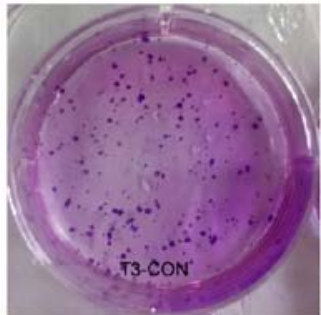

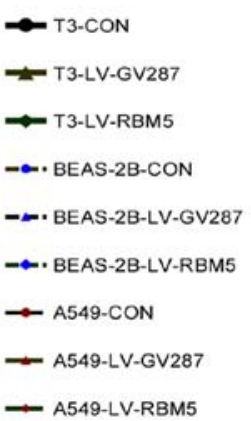

C
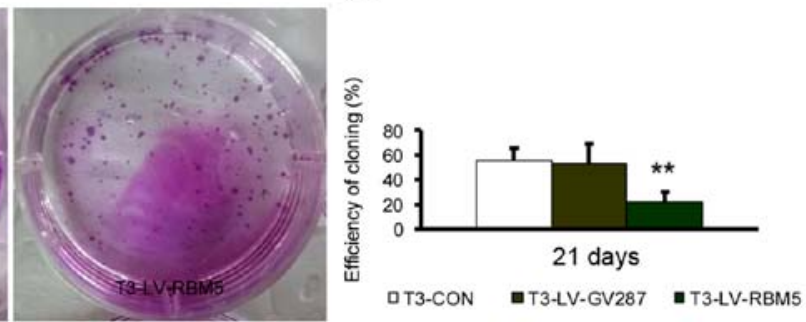

Figure 4. Analysis of the effect of RBM5 overexpression on cell proliferation and colony formation of CSE-transformed BEAS-2B cells (T3 cells). (A) The proliferation following the induced overexpression of RBM5 in the CSE-transformed BEAS-2B (T3 cells), A549 and BEAS-2B cells as determined by the MTT assay and shown as an optical density value in the graph. (B) The colony formation efficacy of either RBM5-overexpressing, control vector or uninfected T3 cells. (C) The colony formation efficiency percentage. Data are expressed as the means \pm SD of individual groups of cells from three independent experiments. ${ }^{* *} \mathrm{P}<0.01$, vs. the control.

invasion and migration revealed that RBM5 overexpression decreased the relative levels of HIF-1 $\alpha$, VEGF, MMP-2 and
MMP-9 (Fig. 5E and F). These data demonstrated that RBM5 inhibited the invasion and migration of CSE-transformed 
A

C

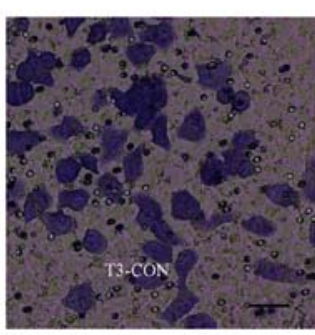

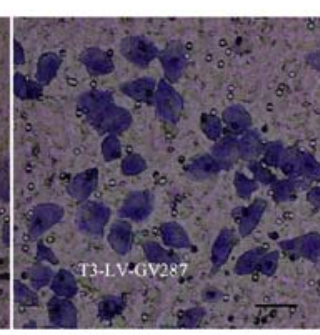

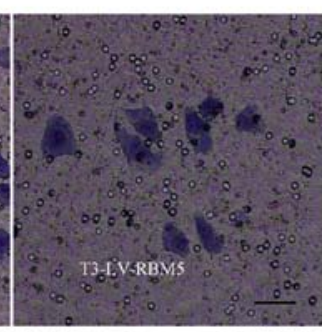

B

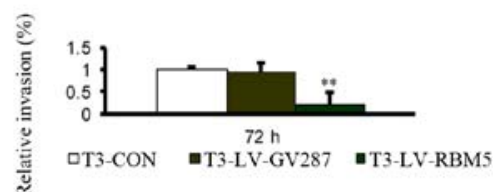

D

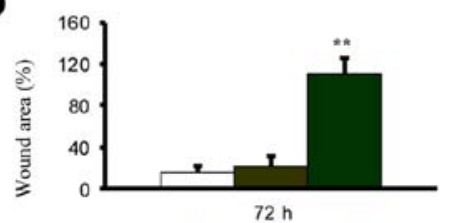

aT3-CON $=$ T3-LV-GV287 $\quad$ =T3-LV-RBM5
$\mathbf{E}$

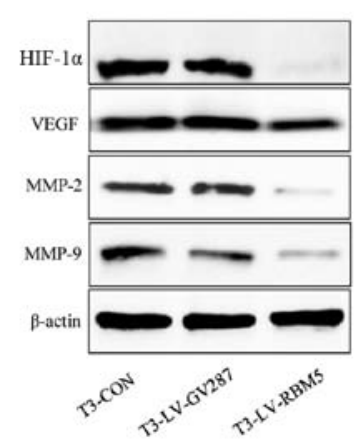

F

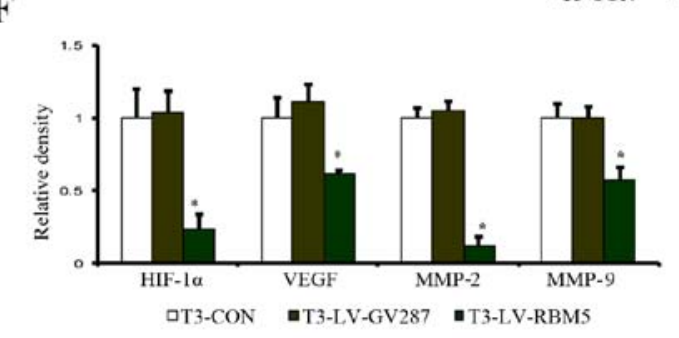

Figure 5. Analysis of the effect of RBM5 overexpression on the invasion and migration of CSE-transformed BEAS-2B cells (T3 cells). (A) The cell invasion efficiency of LV-RBM5, LV-GV287 and the uninfected T3 cells. Scale bar, $20 \mu \mathrm{m}$. (B) Quantitation of the cell invasion percentage. (C) The migration efficiency of the above-mentioned groups of cells. (D) Quantitation of the percentage of the uncovered areas in the wound-healing assays. (E) The protein expression of invasion and migration-related proteins HIF-1 $\alpha$, VEGF, MMP-2 and MMP-9 as determined by western blot assays. (F) Quantitation of the relative densities of these proteins normalized against the internal control $\beta$-actin. Data represent the means \pm SD of individual groups of cells from three independent experiments. ${ }^{*} \mathrm{P}<0.05,{ }^{* *} \mathrm{P}<0.01$ vs. the control.

BEAS-2B cells, most probably by regulating the expression of these pathway-related genes.

RBM5 overexpression induces cell cycle arrest of CSE-transformed BEAS-2B cells at the GI/S phase. In an effort to understand the mechanism of RBM5 overexpression on inhibition of cell proliferation, we first analyzed the effects of RBM5 on the cell cycle distribution in CSE-transformed BEAS-2B cells using flow cytometry. The results revealed that RBM5-overexpressing T3 cells (T3-LV-RBM5) had a significantly increased fraction of cells in the G1 phase and a decreased percentage in the $S$ phase (Fig. 6A). The overall distribution of cells in each phase of the cell cycle among all the groups is shown in Fig. 6B. Further analysis of the expression of cell cycle regulators indicated that the T3-LV-RBM5 cells had significantly decreased relative levels of CDK4, CDK6, cyclin D1 and cyclin A, but increased levels of p53 and p21 (Fig. 6C and D). These data indicate that RBM5 induced cell cycle arrest at the G1/S phase in the CSE-transformed BEAS-2B cells, probably through modulating the expression of cell cycle regulators.

RBM5 overexpression induces apoptosis of CSE-transformed $B E A S-2 B$ cells. To further understand the mechanism of RBM5 in cell proliferation, we also analyzed the effect of RBM5 overexpression on apoptosis by labeling the cells with FITC-Annexin V and PI, followed by flow cytometric analysis. As shown in Fig. 7A, the percentage of apoptotic cells in the T3-LV-RBM5 group was significantly higher than that in the
T3-CON and T3-LV-GV287 groups $(\mathrm{P}<0.05)$. Furthermore, RBM5 overexpression significantly increased the levels of cleaved caspases-3 and -9, along with Bax expression. In contrast, it inhibited the expression of the anti-apoptotic protein Bcl-2 (Fig. 7B and C). Collectively, RBM5 overexpression triggered the apoptosis of CSE-transformed BEAS-2B cells.

RBM5 modulates the relative $m R N A$ levels of many genes implicated in cell invasion, apoptosis and cell cycle regulation. We established that RBM5 overexpression regulated many genes implicated in cell invasion, migration, cell cycle regulation and apoptosis at the protein level. To further identify whether RBM5 overexpression has any effects on these genes at the transcriptional (mRNA) level, we analyzed their relative expression by qRT-PCR, compared against the housekeeping gene GAPDH. The results demonstrated that T3 cells infected with LV-RBM5 had upregulated relative levels of caspase-3, caspase-9 and Bax mRNA transcripts (Fig. 8). In contrast, T3-LV-RBM5 cells had significantly decreased mRNA transcript levels of the anti-apoptotic gene Bcl-2 $(\mathrm{P}<0.05)$. Furthermore, RBM5 overexpression significantly reduced the relative levels of CDK4, CDK6, cyclin D1 and cyclin A, but elevated the levels of p53 and p21 mRNA transcripts $(\mathrm{P}<0.05)$, indicating that RBM5 induced cell cycle arrest in the CSE-transformed BEAS-2B cells. In addition, RBM5 overexpression significantly decreased the relative levels of HIF-1 $\alpha$, VEGF, MMP-2 and MMP-9 expression ( $\mathrm{P}<0.05)$, explaining its effect on the inhibition of invasion and migration of the CSE-transformed BEAS-2B cells. 
A

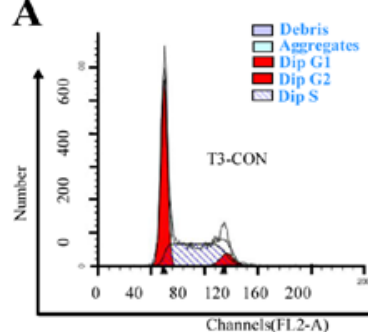

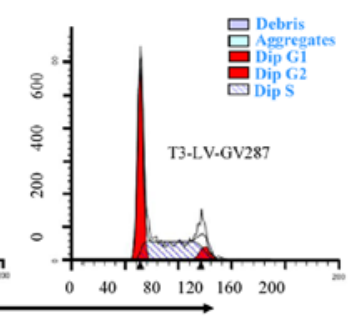

B

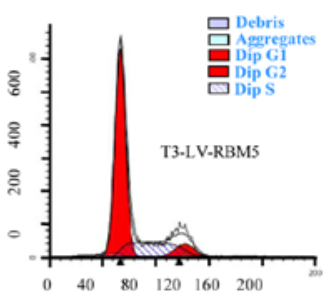

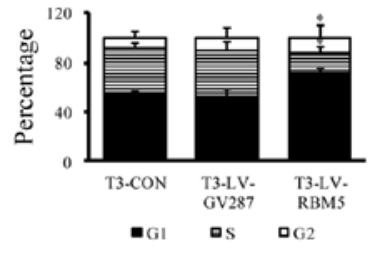

C

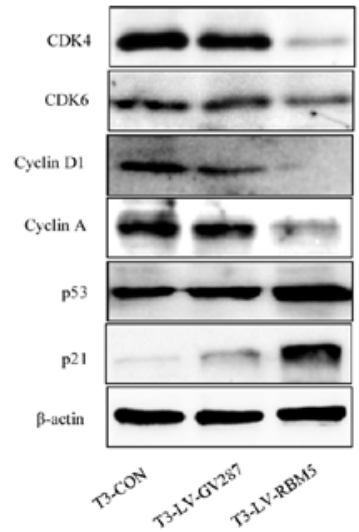

D

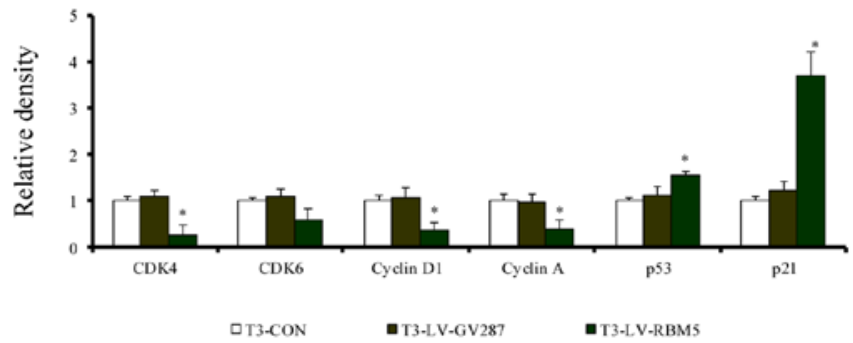

Figure 6. Analysis of the effect of RBM5 overexpression on cell cycle distribution of CSE-transformed BEAS-2B cells (T3 cells). (A) Flow cytometry-based cell cycle analysis of T3 cells either infected with LV-RBM5 or LV-GV287 or not infected. (B) Quantitative representation of the overall cell cycle distribution in the bar graph. (C) Expression of the cell cycle regulators CDK4, CDK6, cyclin D1, cyclin A, p53 and p21, as determined by western blot analysis. (D) Relative densities of these proteins normalized to $\beta$-actin expression. Data are expressed as the means \pm SD of individual groups of cells from three independent experiments. ${ }^{*} \mathrm{P}<0.05$, vs. the control.

A
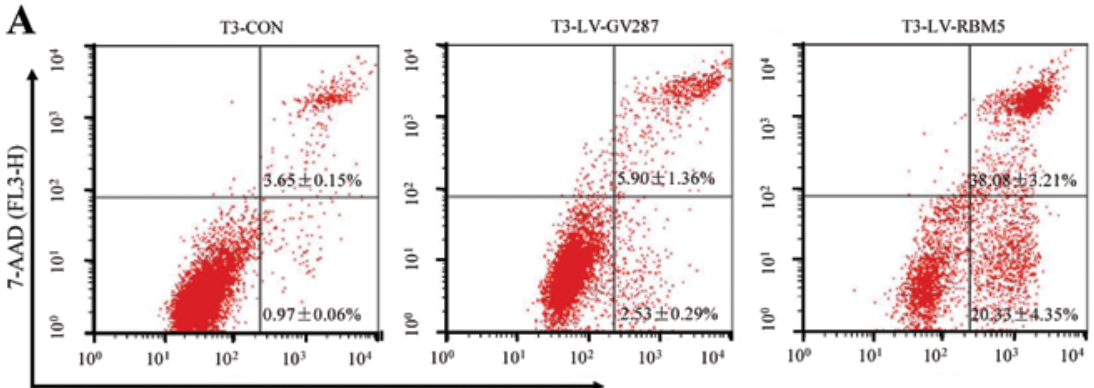

B

C
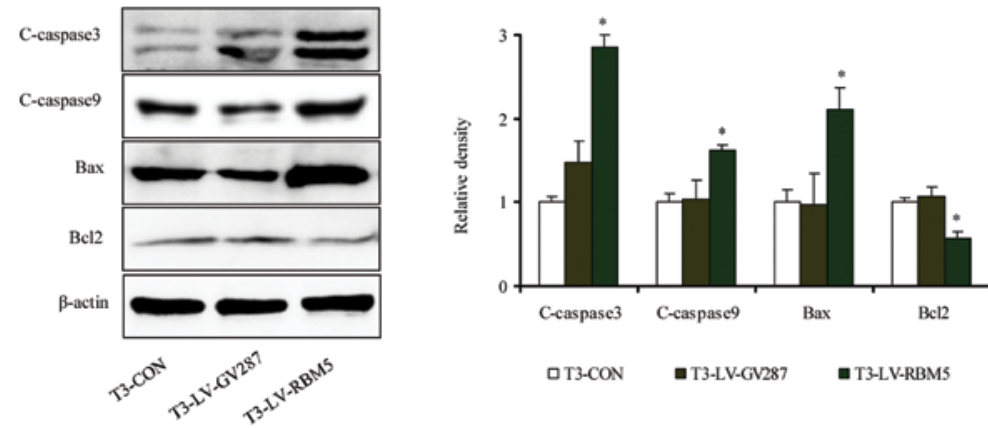

Figure 7. Analysis of the effect of RBM5 overexpression on apoptosis of CSE-transformed BEAS-2B cells (T3 cells). (A) Annexin V-FITC/PI staining ratios as analyzed by flow cytometry of the T3 cells infected with either LV-RBM5 or LV-GV287, or no infection. (B) Protein expression analysis of the apoptosisrelated genes cleaved caspase-3 (C-caspase-3), cleaved caspase-9 (C-caspase-9), Bax and Bcl-2. The $\beta$-actin expression was used as an internal control. (C) Quantitative expression of these proteins after normalization with $\beta$-actin expression. Data points represent the mean \pm SD of individual groups of cells from three independent experiments. ${ }^{*} \mathrm{P}<0.05$, vs. the control. 


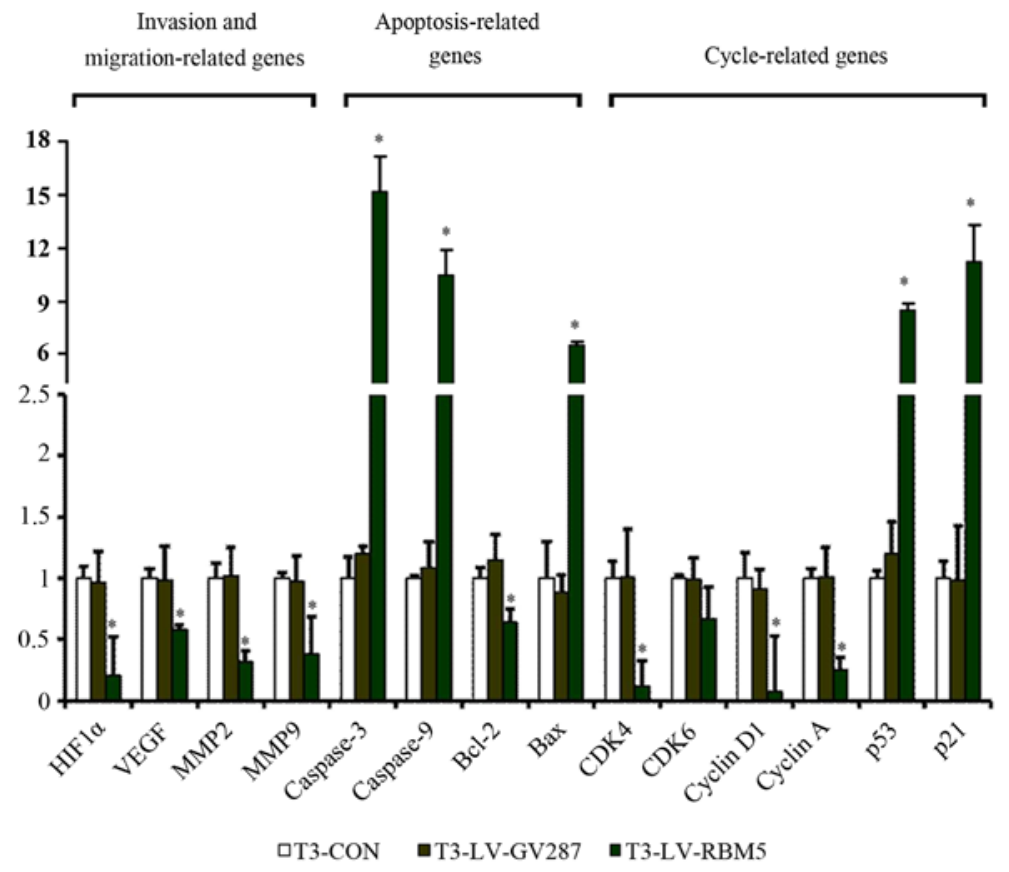

Figure 8. Analysis of the effect of RBM5 overexpression on the mRNA levels of regulatory genes in the CSE-transformed BEAS-2B cells. The T3 cells either infected with LV-RBM5 or LV-GV287, or uninfected were analyzed by RT-PCR to determine the mRNA levels of the regulatory genes associated with invasion, migration, apoptosis and cell cycle regulation. Data are expressed as the means \pm SD of individual groups of cells from three independent experiments. ${ }^{*} \mathrm{P}<0.05,{ }^{* *} \mathrm{P}<0.01$ vs. the control.

A

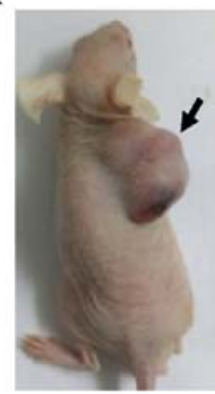

T3-CON

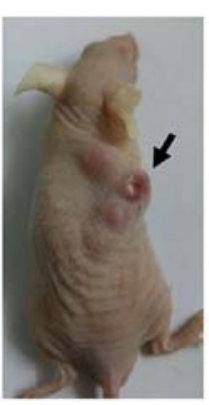

T3-LV-GV287

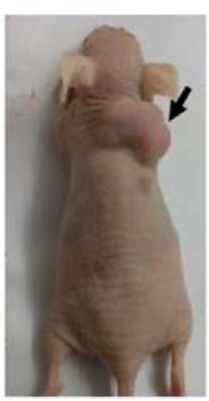

T3-LV-RBM5
B

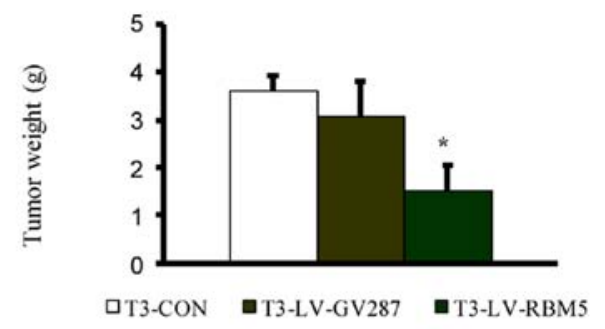

C

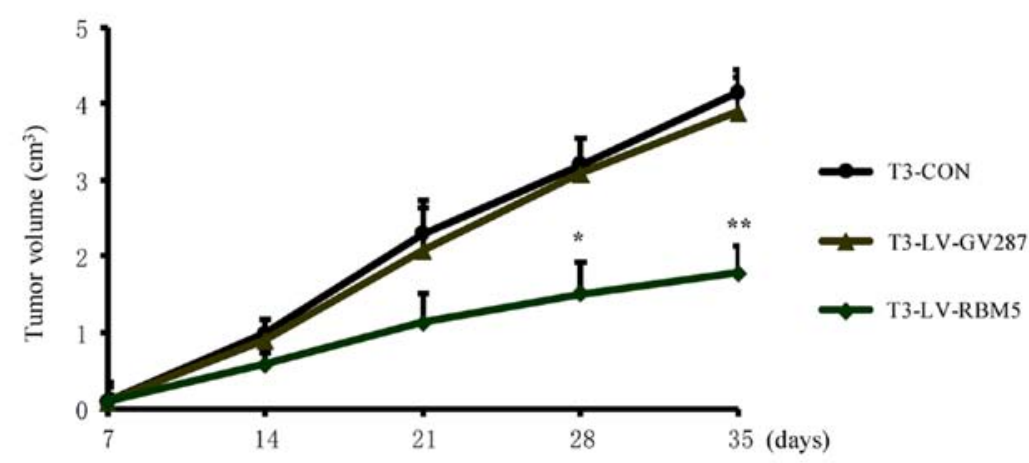

Figure 9. Analysis of the effects of RBM5 overexpression on the xenograft growth of CSE-transformed BEAS-2B cells (T3 cells). (A) Representative images of tumor formation in nude mice ( $\mathrm{n}=6$ for each cell line) and the size of the resulting tumors from T3 cells infected either with LV-RBM5 or LV-GV287, or not infected (T3-CON). (B) Tumor weight of the tumors after sacrificing the nude mice after 4 weeks. (C) Tumor volume of these tumors after subcutaneous injection into the nude mice after 4 weeks. ${ }^{*} \mathrm{P}<0.05,{ }^{* *} \mathrm{P}<0.01$ vs. the control group.

LV-RBM5 resulted in a 55 and $50 \%$ reduction in tumor weight and volume, respectively, measured after 4 weeks, compared to the control groups (Fig. 9). Thus, these data indicate that RBM5 overexpression significantly inhibited xenograft growth. 


\section{Discussion}

In the present study, we established a cellular model of continual CSE exposure of human non-tumorous bronchial epithelial cells and showed that CSE can damage bronchial epithelial cells by inducing hyperplasic growth, malignant cell transformation and tumorigenesis. We observed that BEAS-2B cells surviving 8 days of repeated CSE exposure, followed by a recovery period of 2 weeks, were endowed with phenotypic changes that were characteristic of oncogenic transformation, including increased cell proliferation, enhanced invasion and migration activity, and more importantly, the ability to promote xenograft growth. Similarly, at the cellular level, we observed upregulation of the expression of certain oncogenes, including c-myc, K-ras, cyclin A and cyclin D1 in the transformed BEAS-2B cells. Our results revealing enhancement of the neoplastic transformation of BEAS-2B cells by prolonged treatment with CSE were consistent with previously published data (20). Meanwhile, analysis of RBM5 expression in these transformed cells revealed that it was downregulated at both the mRNA and protein levels and was equivalent to the expression in another tumor cell line (A549), which was analyzed as a control. This observation led us to believe that RBM5 expression may be negatively correlated with smoking-related lung cancer.

To further understand the correlation between RBM5 overexpression and cigarette smoke-induced cancerous transformation of bronchial epithelial cells, we overexpressed wild-type RBM5 using the recombinant lentiviral vector LV-RBM5 into the transformed BEAS-2B cells. Further analysis of these transformed cells revealed that RBM5 overexpression significantly inhibited their cell proliferation and ability to invade and migrate along with their colony-forming potential. It also induced G1/S arrest and apoptosis. In addition, the xenograft growth of transformed BEAS-2B cells was inhibited as a result of RBM5 overexpression. The RBM5 overexpression resulted in inhibition of cell cycle regulatory genes, including CDK4, CDK6, cyclin A and cyclin D1, while the expression of $\mathrm{p} 53$ and $\mathrm{p} 21$ was increased. Similarly, the analysis of apoptosis-related genes (caspase-3 and caspase-9, Bax and $\mathrm{Bcl}-2$ ) revealed that RBM5 overexpression significantly altered their expression. The levels of cleaved caspase- 3 and caspase- 9 as well as Bax expression were increased, whereas the expression level of the anti-apoptotic gene Bcl-2 was decreased. Moreover, we analyzed the expression of regulatory genes (HIF-1 $\alpha$, VEGF, MMP-2 and MMP-9) associated with cell migration and invasion and observed that their expression was significantly downregulated by RBM5 overexpression. Thus, our study demonstrated that RBM5 overexpression inhibited the proliferation of CSE-transformed BEAS-2B cells through regulation of cell cycle arrest and apoptosis by modulating the expression of key genes involved in both of these pathways.

RBM5 is a nuclear RNA-binding protein that is widely distributed in mammalian tissues (21). Accumulating evidence suggests that RBM5 functions as a tumor-suppressor protein by inhibiting tumor transformation and progression (22). The expression of RBM5 mRNA transcript and protein has been observed to be decreased in $70-80 \%$ of lung cancers (8). Despite the increasing evidence suggesting that RBM5 downregulation plays an important role in lung cancer initiation, progression, metastasis and drug resistance $(4,8,12,23)$, its functional mechanism remains unclear. Our studies on the antitumor mechanisms of RBM5 were mostly focused on its role in the cell cycle and apoptosis. Another previous study in leukemic cells showed the involvement of RBM5 overexpression in inhibiting cell proliferation by extending the G1 phase of the cell cycle (24). RBM5 has also been suggested to inhibit both in vitro and in vivo tumor growth of lung cancer cells, with antitumor mechanisms involving G1 cell cycle arrest (5). The normal cell progression from the G1-S-G2-M-G0 phases during the cell division cycle is tightly controlled by the protein kinase activity of a class of serine-threonine kinases (CDKs). CDK4 and CDK6, called the cyclin-dependent kinases, as well as cyclin A and cyclin D1 are important factors during normal cell progression from G1-S-G2-M-G0 (25-27). The G1/S phase transition is negatively regulated by p21 and p27 through the degradation of cyclin/CDK complexes $(28,29)$. In addition, p53 is an important regulator of p21 expression (30). Thus, based on our data we speculated that overexpression of RBM5 would upregulate $\mathrm{p} 53$ expression, which in turn would elevate the expression of p21. This $\mathrm{p} 21$ expression would then subsequently inhibit the cyclin/CDK complex, eventually leading to cell cycle arrest at the G1/S phase in CSE-transformed BEAS-2B cells.

It has been previously suggested that RBM5 modulates apoptosis by regulating the alternative splicing of apoptosis-associated pre-mRNAs, such as CASP2 and FAS/CD95 (12,31). Increased levels of RBM5 promote apoptosis through several pathways, including Fas, TNF- $\alpha$, TRAIL and p53-mediated apoptosis $(32,33)$. Induction of apoptosis by RBM5 has also been correlated with altered levels of Bcl-2, Bcl-x, and Bax as well as activation of cleaved caspases-9 and $-3(4,16,34)$. In addition, it has been suggested that upregulation or downregulation of RBM5 causes changes in the transcription levels of $\sim 35$ genes known to control cell proliferation and apoptosis (17). Our results were consistent with these previous findings in lung cancer and further support the notion that RBM5 may function as a tumor suppressor by promoting the induction of apoptosis in CSE-transformed BEAS-2B cells.

The progression of cancer to different sites largely involves the ability of cancer cells to invade and migrate. The inhibition of these pathways is essentially one of the strategies to combat cancer (35). The migration and invasion of cancer cells are regulated by many factors, such as chemokines, their receptors, the epithelial-mesenchymal transition process, matrix-degrading and antioxidant enzymes, such as MMPs, which mediate the degradation of extracellular matrix proteins $(36,37)$. Among these proteases, MMP-2 and MMP-9 are the most prominent. Furthermore, the migration and invasion can also be modulated by hypoxic conditions and angiogenesis regulators, including HIF-1 $\alpha$ and VEGF $(38,39)$. In the present study, we identified that RBM5 overexpression significantly inhibited the migration and invasion of CSE-transformed BEAS-2B cells by significantly attenuating the expression of HIF- $1 \alpha$, VEGF, MMP-2 and MMP-9 proteins.

In conclusion, we demonstrated that RBM5 expression was inhibited in CSE-transformed BEAS-2B cells and that subsequent overexpression of RBM5 in these cells significantly inhibited the proliferation of cigarette smoke-induced 
transformed BEAS-2B cells through induction of cell cycle arrest and apoptosis. Furthermore, RBM5 overexpression also reduced the xenograft growth of CSE-transformed BEAS-2B cells. Therefore, we hypothesized that RBM5 may be a promising candidate for intervention of smoking-related lung cancer, and our findings may provide new insights into the understanding of the precise mechanism underlying the antitumor activity of RBM5.

\section{Acknowledgements}

The present study was supported by grants from the National Natural Science Foundation of China (nos. 81472169 and 81241069).

\section{References}

1. Chen M, Yang T, Meng X and Sun T: Azithromycin attenuates cigarette smoke extract-induced oxidative stress injury in human alveolar epithelial cells. Mol Med Rep 11: 3414-3422, 2015.

2. Lemjabbar-Alaoui H, Dasari V, Sidhu SS, Mengistab A, Finkbeiner W, Gallup M and Basbaum C: Wnt and Hedgehog are critical mediators of cigarette smoke-induced lung cancer. PLoS One 1: e93, 2006.

3. Sasco AJ, Secretan MB and Straif K: Tobacco smoking and cancer: A brief review of recent epidemiological evidence. Lung Cancer 45 (Suppl 2): S3-S9, 2004.

4. Oh JJ, Razfar A, Delgado I, Reed RA, Malkina A, Boctor B and Slamon DJ: 3p21.3 tumor suppressor gene H37/Luca15/RBM5 inhibits growth of human lung cancer cells through cell cycle arrest and apoptosis. Cancer Res 66: 3419-3427, 2006.

5. Mourtada-Maarabouni M, Sutherland LC, Meredith JM and Williams GT: Simultaneous acceleration of the cell cycle and suppression of apoptosis by splice variant delta- 6 of the candidate tumour suppressor LUCA-15/RBM5. Genes Cells 8: 109-119, 2003.

6. Zhao L, Li R, Shao C, Li P, Liu J and Wang K: 3p21.3 tumor suppressor gene RBM5 inhibits growth of human prostate cancer PC-3 cells through apoptosis. World J Surg Oncol 10: 247, 2012.

7. Welling DB, Lasak JM, Akhmametyeva E, Ghaheri B and Chang LS: cDNA microarray analysis of vestibular schwannomas. Otol Neurotol 23: 736-748, 2002.

8. Oh JJ, West AR, Fishbein MC and Slamon DJ: A candidate tumor suppressor gene, $H 37$, from the human lung cancer tumor suppressor locus 3p21.3. Cancer Res 62: 3207-3213, 2002.

9. Rintala-Maki ND, Goard CA, Langdon CE, Wall VE, Traulsen KE, Morin CD, Bonin $M$ and Sutherland LC: Expression of RBM5-related factors in primary breast tissue. $\mathrm{J}$ Cell Biochem 100: 1440-1458, 2007.

10. Oh JJ, Grosshans DR, Wong SG and Slamon DJ: Identification of differentially expressed genes associated with HER-2/neu overexpression in human breast cancer cells. Nucleic Acids Res 27: 4008-4017, 1999.

11. Wang W, Cassidy J, O'Brien V, Ryan KM and Collie-Duguid E: Mechanistic and predictive profiling of 5-fluorouracil resistance in human cancer cells. Cancer Res 64: 8167-8176, 2004.

12. Sutherland LC, Wang K and Robinson AG: RBM5 as a putative tumor suppressor gene for lung cancer. J Thorac Oncol 5: 294-298, 2010

13. Oh JJ, Taschereau EO, Koegel AK, Ginther CL, Rotow JK, Isfahani KZ and Slamon DJ: RBM5/H37 tumor suppressor, located at the lung cancer hot spot 3p21.3, alters expression of genes involved in metastasis. Lung Cancer 70: 253-262, 2010.

14. Akhtar MJ, Ahamed M, Khan MA, Alrokayan SA, Ahmad I and Kumar S: Cytotoxicity and apoptosis induction by nanoscale talc particles from two different geographical regions in human lung epithelial cells. Environ Toxicol 29: 394-406, 2014.

15. Niu Z, Jin W, Zhang L and Li X: Tumor suppressor RBM5 directly interacts with the DExD/H-box protein DHX15 and stimulates its helicase activity. FEBS Lett 586: 977-983, 2012.

16. Mourtada-Maarabouni M, Sutherland LC and Williams GT: Candidate tumour suppressor LUCA-15 can regulate multiple apoptotic pathways. Apoptosis 7: 421-432, 2002.
17. Mourtada-Maarabouni M, Keen J, Clark J, Cooper CS and Williams GT: Candidate tumor suppressor LUCA-15/RBM5/H37 modulates expression of apoptosis and cell cycle genes. Exp Cell Res 312: 1745-1752, 2006

18. Bechara EG, Sebestyén E, Bernardis I, Eyras E and Valcárcel J: RBM5, 6, and 10 differentially regulate NUMB alternative splicing to control cancer cell proliferation. Mol Cell 52: 720-733, 2013.

19. Schamberger AC, Mise N, Jia J, Genoyer E, Yildirim AÖ, Meiners $S$ and Eickelberg O: Cigarette smoke-induced disruption of bronchial epithelial tight junctions is prevented by transforming growth factor- $\beta$. Am J Respir Cell Mol Biol 50: 1040-1052, 2014

20. Du H, Sun J, Chen Z, Nie J, Tong J and Li J: Cigarette smokeinduced failure of apoptosis resulting in enhanced neoplastic transformation in human bronchial epithelial cells. J Toxicol Environ Health A 75: 707-720, 2012.

21. Drabkin HA, West JD, Hotfilder M, Heng YM, Erickson P, Calvo R, Dalmau J, Gemmill RM and Sablitzky F: DEF-3 (g16/NY-LU-12), an RNA binding protein from the 3p21.3 homozygous deletion region in SCLC. Oncogene 18: 2589-2597, 1999.

22. Maarabouni MM and Williams GT: The antiapoptotic RBM5/ LUCA-15/H37 gene and its role in apoptosis and human cancer: Research update. ScientificWorldJournal 6: 1705-1712, 2006.

23. Liang H, Zhang J, Shao C, Zhao L, Xu W, Sutherland LC and Wang K: Differential expression of RBM5, EGFR and KRAS mRNA and protein in non-small cell lung cancer tissues. J Exp Clin Cancer Res 31: 36, 2012.

24. Kobayashi T, Ishida J, Musashi M, Ota S, Yoshida T, Shimizu Y, Chuma M, Kawakami H, Asaka M, Tanaka J, et al: p53 transactivation is involved in the antiproliferative activity of the putative tumor suppressor RBM5. Int J Cancer 128: 304-318, 2011.

25. Satyanarayana A and Kaldis P: Mammalian cell-cycle regulation: Several Cdks, numerous cyclins and diverse compensatory mechanisms. Oncogene 28: 2925-2939, 2009.

26. Hochegger H, Takeda S and Hunt T: Cyclin-dependent kinases and cell-cycle transitions: Does one fit all? Nat Rev Mol Cell Biol 9: 910-916, 2008

27. Fung TK and Poon RY: A roller coaster ride with the mitotic cyclins. Semin Cell Dev Biol 16: 335-342, 2005.

28. Hedberg Y,Ljungberg B, Roos G and Landberg G: Retinoblastoma protein in human renal cell carcinoma in relation to alterations in G1/S regulatory proteins. Int J Cancer 109: 189-193, 2004.

29. Vermeulen K, Van Bockstaele DR and Berneman ZN: The cell cycle: A review of regulation, deregulation and therapeutic targets in cancer. Cell Prolif 36: 131-149, 2003.

30. Lv XJ, Zhao LJ, Hao YQ, Su ZZ, Li JY, Du YW and Zhang J: Schisandrin B inhibits the proliferation of human lung adenocarcinoma A549 cells by inducing cycle arrest and apoptosis. Int J Clin Exp Med 8: 6926-6936, 2015.

31. Fushimi K, Ray P, Kar A, Wang L, Sutherland LC and Wu JY: Up-regulation of the proapoptotic caspase 2 splicing isoform by a candidate tumor suppressor, RBM5. Proc Natl Acad Sci USA 105: 15708-15713, 2008

32. Rintala-Maki ND and Sutherland LC: LUCA-15/RBM5, a putative tumour suppressor, enhances multiple receptor-initiated death signals. Apoptosis 9: 475-484, 2004.

33. Rintala-Maki ND, Abrasonis V, Burd M and Sutherland LC: Genetic instability of RBM5/LUCA-15/H37 in MCF-7 breast carcinoma sublines may affect susceptibility to apoptosis. Cell Biochem Funct 22: 307-313, 2004.

34. Sutherland LC, Lerman M, Williams GT and Miller BA: LUCA-15 suppresses CD95-mediated apoptosis in Jurkat T cells. Oncogene 20: 2713-2719, 2001

35. Sleeman J and Steeg PS: Cancer metastasis as a therapeutic target. Eur J Cancer 46: 1177-1180, 2010.

36. Verma S, Kesh K, Ganguly N, Jana S and Swarnakar S: Matrix metalloproteinases and gastrointestinal cancers: Impacts of dietary antioxidants. World J Biol Chem 5: 355-376, 2014.

37. Gencer S, Cebeci A and Irmak-Yazicioglu MB: Matrix metalloproteinase gene expressions might be oxidative stress targets in gastric cancer cell lines. Chin J Cancer Res 25: 322-333, 2013.

38. Ramakrishnan S, Subramanian IV, Yokoyama Y and Geller M: Angiogenesis in normal and neoplastic ovaries. Angiogenesis 8: $169-182,2005$

39. Yamakuchi M, Lotterman CD, Bao C, Hruban RH, Karim B, Mendell JT, Huso D and Lowenstein CJ: P53-induced microRNA-107 inhibits HIF-1 and tumor angiogenesis. Proc Natl Acad Sci USA 107: 6334-6339, 2010. 